\title{
Neutrophil to Lymphocyte Ratio (NLR) at the Time of Transurethral Resection of Bladder Tumor: A Large Retrospective Study and Analysis of Racial Differences
}

\author{
Ngii N. Tazeh ${ }^{\mathrm{a}}$, Daniel J. Canter ${ }^{\mathrm{b}}$, Shivashankar Damodaran ${ }^{\mathrm{a}}$, Timothy Rushmer ${ }^{\mathrm{a}}$, Kyle A. Richards ${ }^{\mathrm{a}}$, \\ E. Jason Abel ${ }^{\mathrm{a}}$, David F. Jarrard ${ }^{\mathrm{a}}$ and Tracy M. Downs ${ }^{\mathrm{a}, *}$ \\ ${ }^{a}$ Department of Urology, University of Wisconsin Hospital and Clinics, Madison, WI, USA \\ ${ }^{\mathrm{b}}$ Oschner Medical Center, New Orleans, LA, USA
}

\begin{abstract}
.
Introduction: Neutrophil/lymphocyte ratio (NLR) is an indicator of systemic inflammation and has been proven to be associated with an increased risk of extravesical disease, decreased cancer specific survival and overall survival in bladder cancer patients. A large proportion of healthy African Americans have a WBC count that is persistently lower than the normal range defined for individuals of European ancestry, this condition has been called "benign ethnic neutropenia". The purpose of our study was to determine if NLR was different in patients of African ancestry (AA) vs European ancestry (EA) across different tumor grades and stages at the time of transurethral resection of bladder tumor(s) (TURBT).

Materials and Methods: The records of consecutive patients who underwent TURBT were reviewed from the University of Wisconsin and the Atlanta Veterans' Administration Medical Center (2000-2012). NLR was compared across tumor stage, tumor grade and ethnicity.

Results: 297 consecutive patients met study criteria. 89\% and 86\%, were males and of European ancestry (EA) respectively. NLRs were different across T-stages (Ta-2.5, T1-3.9, T2-3.8; $p=0.001$ ). but not across tumor grades in Ta (LG-2.5 vs HG-3.9, $p=0.57)$. EA had higher NLRs than AA (3.4 vs $1.9 ; p<0.001)$.

Conclusions: Higher NLRs appear to be associated with more advanced tumor stage at the time of TURBT. Patients of African ancestry have lower NLRs across all tumor stages compared to patients of European ancestry. Ethnicity should be taken into account when interpreting the NLR in patients with bladder cancer.
\end{abstract}

Keywords: Bladder cancer, transurethral resection, ethnicity, prognosis

\section{ABBREVIATIONS}

BEN

Benign Ethnic Neutropenia

NLR Neutrophil/Lymphocyte Ratio

UWHC University of Wisconsin Hospital and Clinics

\footnotetext{
*Correspondence to: Tracy M. Downs, MD, FACS, Associate Professor of Urologic Oncology, Department of Urology, Assistant Dean of Diversity and Multicultural Affairs, University of Wisconsin School of Medicine and Public Health, 1685 Highland Avenue, Madison, WI 53705, USA. Tel.: +1 608263 9534; Fax: +1 608262 6453; E-mail: downs@urology.wisc.edu.
}

$\begin{array}{ll}\text { VAMC } & \text { Veterans Affairs Medical Center } \\ \text { AA } & \text { African Ancestry } \\ \text { EA } & \text { European Ancestry }\end{array}$

\section{BACKGROUND}

There continues to be a challenge in deciding what the optimal management of bladder cancer is, especially with respect to deciding which patients would likely benefit from neoadjuvant therapies. Prognostic factors have been developed with 
the intention of better risk stratification. These factors include hydronephrosis, tumor size and multifocality, pathologic stage, lymph node metastases and lymphovascular invasion. Most of these factors are only available after radical cystectomy. There continues to be a need for new preoperative prognostic factors to aid in clinical decision making and for patient counseling.

Several studies have investigated the effect of inflammation on carcinogenesis given that cytokines and other inflammatory mediators have been shown to promote angiogenesis and tumor cell metastasis [1-3]. Several inflammatory markers, such as $\mathrm{C}$ reactive protein and Glasgow prognostic score have been suggested as surrogates for biological characteristics in some types of tumors $[4,5]$. The neutrophil/lymphocyte ratio (NLR) is a biomarker of inflammation and an elevated NLR has been shown to be an independent poor prognosticator of overall and disease-specific survival in several malignancies, including colorectal, ovarian, lung, pancreatic, biliary, gastric, renal and bladder cancer [6-9]. Multivariate analyses by Gondo et al. recently found an elevated NLR to be an independent prognostic factor for disease-specific survival in bladder cancer prior to radical cystectomy [10]. NLR is a simple and reproducible laboratory index that is readily available across most practices. "Benign ethnic neutropenia" (BEN) is an interesting finding in the natural biology of neutrophils and refers to a persistently lower neutrophil count noted in $40-50 \%$ of individuals of African ancestry who are otherwise healthy (and other racial groups as well) compared to the normal range as defined for individuals of European ancestry [11]. Reich et al. through admixture genomic scanning were able to map this genetic variant to a single nucleotide substitution polymorphism called FY+/- (rs2814778) or "Duffy" variant located on the long arm of chromosome 1 [12]. This polymorphic phenotype is known to confer innate resistance to Plasmodium vivax malaria by decreasing RBC infectivity $[13,14]$ and associated with increased susceptibility to HIV infection and progression to AIDS [15]. It is also implicated in the biology of sickle cell disease in West African population [16].

In the present study, we sought to investigate whether African Ancestry (AA) patients in our study cohort had BEN contributing to a proportionately decreased NLR and analyze the associations between NLR and tumor stage and grade within and between ethnic lineages.

\section{MATERIALS AND METHODS}

\section{Study design}

This retrospective study included data on 297 consecutive patients who underwent transurethral resection of bladder tumor(s) (TURBT) as part of their work up for bladder cancer. These patients were seen and evaluated in the departments of urology at the University of Wisconsin Hospital and Clinics (UWHC) as well as the Atlanta Veterans' Affairs Medical Center (VAMC) between 2000 and 2012. Only patients with primary bladder tumor and those who had a complete blood count and differentials prior to TUR were included in the study. NLRs were calculated by dividing neutrophil percentage by the percentage of lymphocytes measured in the differential of a CBC. None of the patients had undergone any prior intravesical therapies. No patient had any evidence of active infection or hematopoietic malignancy at the time of tumor resection. Patients that self-identified as African American were assigned the designation of African Ancestry (AA) in this study, while those that self-described as Caucasian were listed as European Ancestry (EA). Histological evaluation of the specimens was performed at the respective centers mentioned above and TNM staging and grading assigned according to the 2010 American Joint Committee on Cancer/UICC TNM Classification, 7th edition [17]. Other patient demographic details that were retrospectively extracted from the medical records included patient age, sex, and gender. The main outcome measure of this study was tumor grade and stage differences within and across racial lineages. The respective Institutional Review Boards of UWHC and VAMC Atlanta approved this study.

\section{Statistical analysis}

Median and interquartile range of patient characteristics and hematological parameters were compared between the 2 cohorts. Mann Whitney $\mathrm{U}$ test was used to analyze difference between the 2 cohorts. Kruskal-Wallis test was used for computing differences in NLR between T stages and grade stratified clinical stages. Then we calculated the difference in NLR between the individual grade stratified T stage across EA and AA cohorts. All $p$ values were 2 tailed and a value of $<0.05$ was considered statistically significant. 


\section{RESULTS}

\section{Patient characteristics}

Through a retrospective review of the medical records between 2000 and 2012, 297 patients with bladder cancer who underwent TURBT for the first time, at the above centers and met study inclusion criteria were identified. Mean age, BMI, and WBC were 66.7 years, $28.5 \mathrm{~kg} / \mathrm{m} 2$, and $8.1 .41 \%, 22 \%$, and $37 \%$ of patients had Ta, $\mathrm{T} 1$, and $\mathrm{T} 2$ tumor stage at TURBT. There were $255(86 \%)$ men and $42(14 \%)$ women. Forty-two (14\%) AA men were included in this study. The median age of our patient cohort was 66.7 years (IQR 60.1-75.2) with a median WBC count of 7.5 (6.1-9.0). The individual patient characteristics and hematological parameters of both the cohorts are summarized in Table 1.

\section{NLR and bladder cancer grade/stage}

The distribution of NLR's of all the patients in our cohort across different bladder cancer tumor stages is shown in Table 2. Comparison was also done between high and low grade Ta. The NLR ratio was noted to increase with an increase in tumor stage from Ta to $\mathrm{T} 2,(p=0.001)$. In addition, NLR increased between low and high grade of Ta, though this difference was not statistically significant, $(p=0.57)$.

\section{NLR and racial differences across bladder cancer stage and grade}

The median NLR of the AA cohort of our patient population was 1.7 (1.36-2.7), which was lower compared to the EA cohort median of 2.5 (1.81-3.33), $(p=0.006)$. NLR increased with an increase in tumor stage in both EA and AA cohort. (See Table 3) Within the AA cohort the NLR increased overall with tumor stage with medians of 1.7 (1.21-2.22), 1.6
Table 2

NLR means comparison across $T$ stages and grade

\begin{tabular}{|c|c|c|c|c|}
\hline Stage & & NLR mean $(n)$ & Median [IQR] & $p$ value \\
\hline Тa & & 2.5 & $2.2[1.6-2.9]$ & \\
\hline $\mathrm{T} 1$ & & 3.9 & $2.2[1.7-3.4]$ & \\
\hline $\mathrm{T} 2$ & & 3.8 & $3.5[2.2-4.6]$ & 0.001 \\
\hline \multicolumn{5}{|c|}{ NLR was different across $T$ stages. } \\
\hline $\mathrm{Ta}$ & Low & 2.5 & $2.2[1.6-2.9]$ & \\
\hline $\mathrm{Ta}$ & High & 3.9 & $2.4[1.8-2.9]$ & 0.57 \\
\hline \multicolumn{5}{|c|}{ NLR was not different between low and high grade Ta. } \\
\hline
\end{tabular}

(1.44-2.17) and 3.6 (3.06-3.73) for Ta, T1 and T2 disease respectively, $(p<0.01)$. Similarly, the EA cohort showed a corresponding increase in NLR with tumor stage with medians of 2.4 (1.8-3.04), 3.3 (2-3.53) and $3.5(2.06-4.77)$ for Ta, T1 and T2 disease respectively, $(p=0.01)$. In addition, within each tumor stage, AA NLR medians were consistently lower compared with their respective EA counterparts (See Table 3).

When the data for tumor grade was superimposed on the tumor stage data as shown in Table 3, another interesting and consistent finding was evident. Median NLR of high grade Ta was higher compared to low grade Ta, but the difference did not reach statistical significance $(p=0.57)$. Comparison of medians of individual stages of both cohorts is shown in Fig. 1.

Table 3

NLR median with IQR stratified for Stage and Grade, compared between AA and EA cohorts

\begin{tabular}{lcll}
\hline Stage & \multicolumn{1}{c}{ AA } & \multicolumn{1}{c}{ EA } & $p$ Value \\
\hline Ta Low & $1.5(1.12-2.23)$ & $2.4(1.67-2.99)$ & 0.005 \\
Ta High & $1.7(1.47-2.01)$ & $2.4(1.82-2.95)$ & 0.02 \\
T1 High & $1.6(1.45-2.17)$ & $3.3(2-3.53)$ & 0.006 \\
T2 High & $3.6(3.06-3.73)$ & $3.5(2.06-4.77)$ & 0.06 \\
\hline
\end{tabular}

Stage and grade stratified NLR difference was statistically significant between Ta low grade, Ta high grade and T1 high grade tumors.

Table 1

Patient demographics and hematological parameters

\begin{tabular}{lccr}
\hline Variable & AA & EA & $p$ value \\
\hline Number & 42 & 255 & \\
Age & $63(59-72.5)$ & $67(61-76)$ & 0.02 \\
BMI & $26.6(24-31.9)$ & $27(24.46-30.6)$ & 0.72 \\
Charlson comorbidity index & $8(7-10)$ & $8.5(7-10)$ & 0.31 \\
WBC count $\times 1000$ & $7.7(6.2-9.1)$ & $6.8(5.7-8.5)$ & 0.09 \\
Median neutrophil $(\%)$ & $55.9(50.8-65.7)$ & $62.5(56.3-68)$ & $<0.01$ \\
Median lymphocyte $(\%)$ & $32(24.5-37.3)$ & $25.5(20.2-30.2)$ & $<0.01$ \\
Median NLR & $1.7(1.3-2.7)$ & $2.5(1.8-3.3)$ & $<0.01$ \\
\hline
\end{tabular}

Patient demographics and NLR associated parameters (Median/IQR) compared between EA and AA cohorts. 


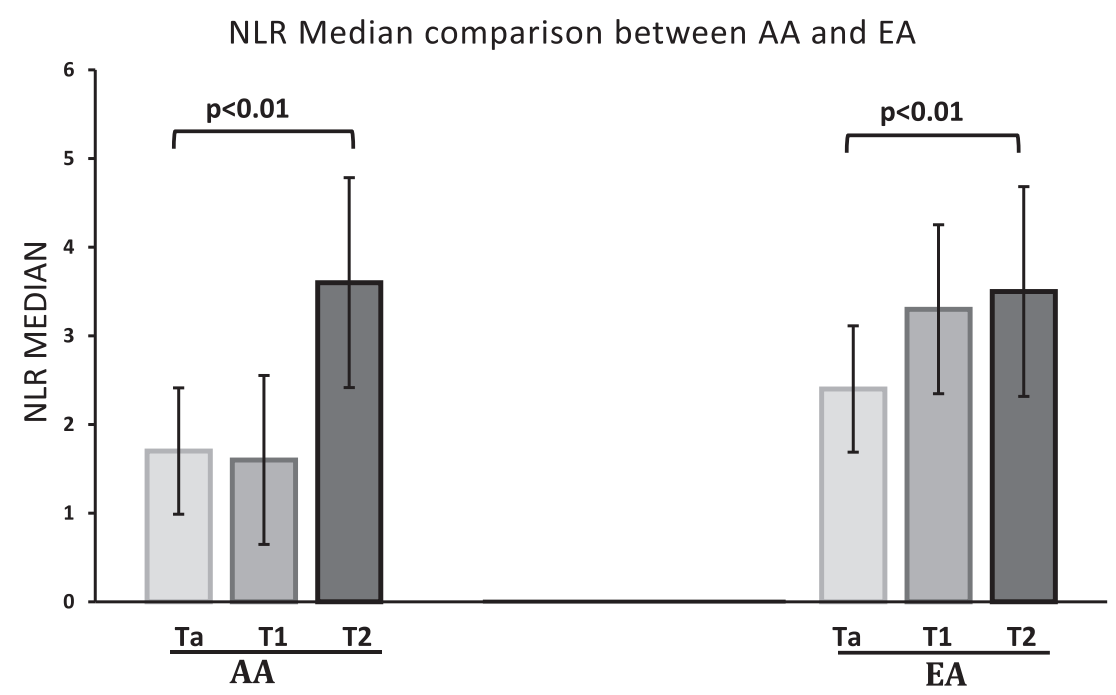

Fig. 1. Median NLR of Ta, T1 and T2 compared between the two ethnic groups. Individual T stage mean NLR compared in AA and EA cohort. The nonparametric variables were compared using Kruskal Wallis test. There was a statistically significant difference between the NLR of Ta, T1 and T2 stage tumors, with $p$ values of $<0.01$ and 0.01 in AA and EA cohorts, respectively.

\section{DISCUSSION}

Tumor development and growth involves interactions with the host stroma and immune modulators [18]. There is a plethora of evidence in the literature showing a linkage between chronic inflammation and tumorigenesis as well as cancer progression $[19,20]$ First, chemokines and cytokines are present in the microenvironment of most tumors in experimental animal models and in humans from the earliest stages of development [2]. Platelets and granulocytes, especially neutrophils, are known to secrete pro-angiogenic factors including vascular endothelial growth factor (VEGF), interleukin 8, matrix metalloproteases and elastases - all known to be involved in tumorigenesis [23]. These factors secreted by neutrophils can help create a micro-environment conducive for tumor development. By extension it is likely that because patients with a high NLR have a relative lymphopenia, they exhibit a poor lymphocyte-mediated response to malignancy, and as a result possibly have worse cancer prognosis. Second, inflammatory diseases and chronic inflammatory conditions are known to increase the risk of developing many types of cancer including colorectal and bladder. Bladder cancer cells produce G-CSF, express G-CSF receptors and stimulate autocrine growth of tumor cells in vivo. Recent reviews in the last decade on the biology of cancer cachexia have recognized the importance of systemic inflammation and have proposed measures of systemic inflammation in their definitions of cancer cachexia $[21,22]$. NLR is a marker of systemic inflammation and a readily available, dynamic variable as well as easily reproducible test can be used as a surrogate marker for cancer cachexia. It has been studied as a useful prognostic biomarker in various cancers such as lung cancer [25] and colorectal cancer [26]. In urologic malignancies, NLR has also been shown to have important prognostic value in predicting clinical outcomes. In a retrospective study by Azuma et al., a NLR cut off of 2.5 was found to be a significant predictor of 5 year cancer specific survival (CSS) $(81.3 \%$ for NLR > 2.5 vs $29.4 \%$ for NLR < 2.5) for upper tract urothelial cancer [27] In another retrospective study in a cohort of invasive bladder cancer undergoing radical cystectomy, Hermanns et al. showed that a NLR $\sim 3.0$ was an independent predictor of recurrence free survival (RFS - HR 1.49), CSS (HR 1.88) and overall survival (OS- HR 1.67) [28]. Similarly in a cohort of advanced urothelial cancer treated with chemotherapy, Rossi et al. showed that a pre chemotherapy and follow up NLR $>3$ had poor progression free survival and median OS [29]. In a meta-analysis of 17 studies including over 3000 patients with urologic cancers, subgroup analysis revealed high NLR to be associated with poor overall survival in renal cell carcinoma (RCC) as well as worse recurrence free survival and cancer specific survival in RCC and urothelial carcinoma [30].

In the present study, we investigated NLR variation across various bladder cancer stages and grades 
as well as how these variations compared across two racial groups. We show that higher NLR ratios were associated with progressively higher bladder cancer stages. This held true within as well as across the two ethnic lineages studied here - African American and European Americans. Neutrophilia and/or relative lymphopenia, hence a high NLR ratio, might promote carcinogenesis and inhibit antitumor immune response, as supported by in vitro [31] studies as well as in melanoma [32]. Our study represents one of the few published works on the potential role of inflammation in bladder cancer tumorigenesis. Gondo et al. recently published their work that showed NLR to have independent prognostic value in bladder cancer prior to radical cystectomy [10]. Their work did not comment on any ethnic variations in the prognostic value of the NLR though it would be reasonable to speculate their cohort was drawn from a very homogenous Asian population. In the meta-analysis by Wei Y et al. [24] a stratified analysis was done based on ethnicity of patients (Asians and Caucasians) and an elevated NLR was found to have different Hazards ratio for Caucasians (HR 1.895 CI 1.33-2.61) and for Asians (HR 2.26 95 CI 1.66-3.09). A recent paper by Mano et al. reported their single-institution experience with 122 patients with newly diagnosed non-muscle invasive bladder cancer who on retrospective review showed high NLR ratios to be an independent predictor of disease progression and recurrence [33]. Using Cox regression analysis, they found an NLR ratios of 2.41 and 2.43 to display a significant dichotomy in outcomes for progression-free survival and time to recurrence respectively, with higher ratios being a poor prognostic factor. The ethnic backgrounds of the subjects in this study were not stated. Nevertheless, these cut-offs are consistent with the ratios revealed in our patient cohort of European Ancestry as reported here where patients with higher risk disease (high grade and/or lamina propria invasion) had mean NLR ratios > 2.4. Interestingly, patients of African Ancestry with high grade Ta and T1 disease had NLR ratios $<2.4$, which re-emphasizes the point that racial background should be taken into consideration when utilizing this ratio as a prognostic measure.

The concept of benign ethnic neutropenia is an intriguing one that brings into play another level of complexity when interpreting NLR ratios. Traditionally, neutropenia irrespective of race has been defined in persons over 1-year-old as an absolute neutrophil count $<1500$ cells/cc [11]. Benign ethnic neutropenia is the phenomenon of neutropenia in otherwise healthy individuals. This has been observed in subjects from various ethnic groups including Africans, Afro-Caribbeans, Yemenite Jews and certain Arab groups. These persons are known to have normal bone marrow morphology with normal numbers of their other blood cell lineages. Through very elegantly designed population-wide admixture mapping studies, Ziv E and others have been able to identify a single substitution nucleotide polymorphism located at chromosome 1q22 locus to be responsible for the differences in white blood cell counts between African Americans and European Americans [11, 12, 24]. This polymorphism is called FY+/- (rs2814778) or "Duffy" variant and has been implicated in conferring resistance to people of West African descent to infection by Plasmodium falciparum as well as in the biology of sickle cell disease and HIV/AIDS. It should therefore be noted that there is an ethnic variation in neutrophil counts that is borne out of a genetic variation that has stood the test of time through eons of evolution. So when using NLR ratios in counseling patients on their prognostic outlook with respect to their specific bladder cancer, racial differences should be taken into consideration.

A limitation of our study is the lack of data on other underlying disease processes or inflammation triggers within our cohort (such as ongoing infection, chronic inflammatory disease as well as exogenous steroids) that could affect the NLR ratio outside of the underlying bladder cancer. The absence of follow up NLR for correlation with clinical outcomes was another limitation of the present study. The retrospective design of this study certainly can inherently introduce some bias into the data analysis. The relatively small number of patients in our study is also another statistical limitation.

In summary, although further validation through independent prospective studies is still needed, the NLR is a readily available and reproducible marker that shows great promise as a new prognostic marker for the aggressive biology of bladder cancer within and across various racial lineages. Potentially this marker can help risk stratify patients prior to TURBT and further large prospective studies are needed to investigate the role of NLR in the pre TURBT setting.

\section{CONFLICT OF INTEREST}

The authors have no conflict to report. 


\section{REFERENCES}

[1] Balkwill F, Mantovani A. Inflammation and cancer: Back to Virchow? Lancet 2001;357:539-45.

[2] Coussens LM, Werb Z. Inflammation and cancer. Nature 2002;420:860-7.

[3] Mantovani A, Allavena P, Sica A, Balkwill F. Cancer-related inflammation. Nature 2008;454:436-44.

[4] Hashimoto K, Ikeda Y, Korenaga D, Tanoue K, Hamatake M, Kawasaki K, Yamaoka T, Iwatani Y, Akazawa K, Takenaka $\mathrm{K}$. The impact of preoperative serum $\mathrm{C}$-reactive protein on the prognosis of patients with hepatocellular carcinoma. Cancer 2005;103:1856-64.

[5] Hefler LA, Concin N, Hofstetter G, Marth C, Mustea A, Sehouli J, Zeillinger R, Leipold H, Lass H, Grimm C, et al. Serum C-reactive protein as independent prognostic variable in patients with ovarian cancer. Clin Cancer Res 2008;14:710-4.

[6] Nakahara Y, Mochiduki Y, Miyamoto Y, Nakahara Y, Katsura Y. Prognostic significance of the lymphocyteto-neutrophil ratio in percutaneous fine-needle aspiration biopsy specimens of advanced non-small cell lung carcinoma. Cancer 2005;104:1271-80.

[7] Shimada H, Takiguchi N, Kainuma O, Soda H, Ikeda A, Cho A, Miyazaki A, Gunji H, Yamamoto H, Nagata M. High preoperative neutrophil-lymphocyte ratio predicts poor survival in patients with gastric cancer. Gastric Cancer 2010;13:170-6.

[8] Chua W, Charles KA, Baracos VE, Clarke SJ. Neutrophil/lymphocyte ratio predicts chemotherapy outcomes in patients with advanced colorectal cancer. Br J Cancer 2011;104:1288-95.

[9] Walsh S, Cook EJ, Goulder F, et al. Neutrophil-Lymphocyte ratio as a prognostic factor in colorectal cancer. J Surg Oncol 2005;91:181-4.

[10] Gondo T, Nakashima J, Ohno Y, et al. Prognostic value of neutrophil-to-lymphocyte ratio and establishment of novel preoperative risk stratification model in bladder cancer patients treated with radical cystectomy. Urology 2012;5:1085-91

[11] Paz Z, Nalls M, Ziv E. The genetics of benign neutropenia. IMAJ 2011;13:625-9.

[12] Reich D, Nalls M, et al. Reduced neutrophil count in people of African descent is due to a regulatory variant in the Duffy antigen receptor for chemokines gene. PLoS Genetics 2009;5:1-14.

[13] Miller L, Mason S, Clyde D, McGinnis M. The resistance factor to Plasmodium vivax in blacks. The Duffy-bloodgroup phenotype, FyFy. NEJM 1976;295:302-4.

[14] Horuk R, Chitnis C, Darbonne W, et al. A receptor for the malarial parasite Plasmodium vivax: The erythrocyte chemokine receptor. Science 1993;261:1182-4.

[15] He W, Neil S, Kulkarni H, et al. Duffy antigen receptor for chemokines mediates trans-infection of HIV-1 from red blood cells to target cells and affects HIV-AIDS susceptibility. Cell Host Microbe 2008;4:52-62.

[16] Afenyi-Annan A, Kail M, Combs MR, Orringer EP, et al. Lack of Duffy antigen expression is associated with organ damage in patients with sickle cell disease. Transfusion 2008;5:917-24.

[17] Edge S, Byrd D, Compton C, et al. The AJCC cancer staging manual. 7th Edition, New York Springer 2010.
[18] Whiteside TL. The tumor microenvironment and its role in promoting tumor growth. Oncogene 2008;27:590412.doi:10.1038/onc.2008.271

[19] Grivennikov SI, Greten FR, Karin M. Immunity, inflammation, and cancer. Cell 2010;140(6):883-99. doi:10.1016/ j.cell.2010.01.025.

[20] Rakoff-Nahoum S. Why cancer and inflammation? The Yale Journal of Biology and Medicine 2006;79(3-4):123-30.

[21] Morley J, Thomas D, Wilson M. Cachexia: Pathophysiology and clinical relevance. Am J Clin Nutr 2006;83:735-43.

[22] Fearon K, Voss A, Hustead D. Cancer cachexia study group. Definition of cancer cachexia: Effect of weight loss, reduced food intake, and systemic inflammation on functional status and prognosis. Am J clin Nutr 2006;83:1345-50.

[23] Wang S, Zhang Z, Fang F, et al. The neutrophil/lymphocyte ratio is an independent prognostic indicator in patients with bone metastasis. Oncology Letters 2011;2:735-40.

[24] Nalls M, Wilson J, Patterson N, et al. Admixture mapping of white cell count: Genetic locus responsible for lower white blood cell count in the Health ABC and Jackson Heart studies. Amer J of Human Genetics 2008;82:81-7.

[25] Cedres S, Torrejon D, Martinez P, et al. Neutrophil to lymphocyte ratio (NLR) as an indicator of poor prognosis in stage IV non-small cell lung cancer. Clin Transl Oncol (Placeholder1) 2012;14:864-9.

[26] He W, Yin C, Guo G, Jiang C, Wang F, et al. Initial neutrophil lymphocyte ratio is superior to Platelet lymphocyte ratio as an adverse prognostic and predictive factor in metastatic colorectal cancer. Med Oncol 2013;30(1):439.

[27] Azuma T, Matayoshi Y, Odani K, Sato Y, Sato Y, Nagase $\mathrm{Y}$, et al. Preoperative neutrophil-lymphocyte ratio as an independent prognostic marker for patients withupper urinary tract urothelial carcinoma. Clin Genitourin Cancer 2013;11(3):337-41. doi: 10.1016/j.clgc.2013.04.003. PubMed PMID: 23665132.

[28] Hermanns T, Bhindi B, Wei Y, Yu J, Noon AP, Richard $\mathrm{PO}$, et al. Pre-treatment neutrophil-to-lymphocyte ratio as predictor of adverse outcomes in patients undergoing radical cystectomy for urothelial carcinoma of the bladder. $\mathrm{Br}$ J Cancer 2014;111(3):444-51. doi:10.1038/bjc.2014.305. PubMed PMID:24918819; PubMed Central PMCID: PMC4119979.

[29] Rossi L, Santoni M, Crabb SJ, Scarpi E, Burattini L, Chau $\mathrm{C}$, et al neutrophil-to-lymphocyte ratio persistent during first-line chemotherapy predicts poor clinical outcome in patients with advanced urothelial cancer. Ann Surg Oncol 2015;22(4):1377-84. doi: 10.1245/s10434-0144097-4. PubMed PMID: 25234022.

[30] Wei Y, Jiang YZ, Qian WH. Prognostic role of NLR in urinary cancers: A meta-analysis. PLoS One 2014;9(3): e92079.

[31] Petrie HT, Klassen LW, Kay HD. Inhibition of human cytotoxic $\mathrm{T}$ lymphocyte activity in vitro by autologous peripheral blood granulocytes. J Immunol 1985;134:230-4.

[32] Schaider H, Oka M, Bogenrieder T, Nesbit M, Satyamoorthy $\mathrm{K}$, et al. Differential response of primary and metastatic melanomas to neutrophils attracted by IL-8. Int J Cancer 2003; 103:335-43.

[33] Mano R, Baniel J, Shoshany O, et al. Neutrophil-tolymphocyte ratio predicts progression and recurrence of non-muscle invasive bladder cancer. Urol Oncol 2015; 33(2):67. 